\title{
Robust Face Recognition Using Distance Matrice
}

\author{
Chirag I. Patel and Ripal Patel
}

\begin{abstract}
In this paper, we have present the face recognition method based on partial Hausdorff distance. Normally face recognition algorithm gives poor results against pose and illumination variation. But the algorithm we have presented is robust to those conditions. We have applied transformation on face image which is robust todifferent face pose and illumination variations. Then the partial Hausdorff distance is calculated for matching after that the performance of face recognition is evaluated on different database.
\end{abstract}

Index Terms-Face recognition, Hausdorff distance, computer vision.

\section{INTRODUCTION}

Hausdorff distance is efficient measure for similarity between two face images. Kwan-Ho Lin et al [1] present face recognition approach based on spatially weighted Hausdorff distance. Eigenface is used as weighting function, which has large value at important facial feature of face structure. Two modified Hausdorff Distance spatially eigenweighted Hausdorff distance (SEWHD) and spatially eigenweighted 'doubly' Hausdorff distance (SEW2HD) are proposed for face recognition. Information about face contour, eye, mouth are incorporated using this approach.

Sanun Srisuk et al [2] proposed new approach for face detection based on enhanced Hausdorff distance. A technique is used to automatic locate face with appropriate size of an elliptical model. Then detection is performed using Hausdorff distance.

Baofeng Gor et al [3] proposed face recognition approach based on modified Hausdorff Distance. The edge map of face images have important information about face and face features. So, Edge maps of faces are considered and Hausdorff Distance is used to measure similarity between that faces.

In [4] survey of face recognition approaches are given with detail description.

In this work, we have implemented a new Partial Hausdorff Distance-based measure to compare the appearance of faces. The measure is applied to face recognition based on gray images of faces instead of edge maps. The measure is robust to variations in a face due to expression, illumination and slight posed differences. The partialness in the measure could tolerate expression and slight pose changes. The transformed face images are less sensitive to illumination changes besides preserving the

Manuscript received January 11, 2013; revised March 14, 2013.

Chirag I. Patel is with the Computer Science Department, Nirma Institute of Technology, Ahmedabad, India (e-mail: chirag453@gmail.com).

Ripal Patel is with the Electronics \& Commu. Department Dharmsinh Desai institute of technology, Nadiad, India (email:ripalpate1315@gmail.com). appearance information of faces. The new measure is defined on these transformed faces. The organization of the paper is as follows. The next section describes the image transformation and shows that the transformation is robust to illumination variations. Section3, define the new Hausdorff distance-based measure, Hpv, being used in this work describes the procedure to calculate it from face recognition and lists the properties of the measure. A time space efficient algorithm is also explained for the computation of Hpv. In section 4,the performance of $\mathrm{Hpv}$ for face recognition is evaluated using benchmark face databases viz. the ORL (AT \& T Laboratories, Cambridge) [5] and the YALE (Yale University, New Haven)[6]. The reference of this work is based on is Vivek/Sudha [7].

\section{IMAGE TRANSFORMATION}

\section{A. Compared Against Edge Map}

Existing Hausdorff distance-based measures for face recognition are denned between edge images of faces. Edge images are less affected by illumination variations. However, edge images do not carry the overall facial appearance. When the gray images that have appearance information are directly considered for comparison, its performance is affected by illumination variations. The effect of illumination can be reduced to a large extent by representing a pixel based on relative intensities of pixels in its neighborhood. Thus, a pixel is represented by a vector rather than a single gray value.

\section{B. Final Stage}

The transformation is carried out by considering the 8neighborhood of the pixel. Consider a pixel and its 8neighborhood which forms a $3 \times 3$ window. The signs of their derivative taken along the direction of neighbors are expected to remain same for uniform illumination changes over the window. The advantage of representing a pixel in terms of its neighborhood is that it captures the distribution of the intensities in the neighborhood.

Each element of vector corresponds to the first derivative along the direction of a neighbor of the pixel. The element takes one of the values, viz. 1,0 and -1 . Let $g(P)$ and $g\left(P_{n}\right)$ are the gray values of pixel $\mathrm{P}$ and its neighbor $\mathrm{P}_{n}$. Then the element (corresponding to $\mathrm{P}_{\mathrm{n}}$ ) of vector assigned to $\mathrm{P}$ takes the value 1 if $g(P)>g\left(P_{n}\right), 0$ if $g(P)=g\left(P_{n}\right)$ and -1 if $g(P)<$ $\mathrm{g}\left(\mathrm{P}_{\mathrm{n}}\right)$. Fig. 1 shows a $3 \times 3$ window in an image and the vector corresponding to pixel $\mathrm{p}$ at centre.

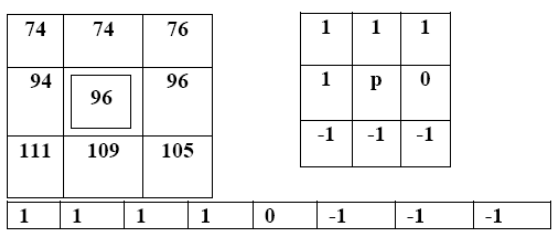

Fig. 1. Transformation to vector image 


\section{Robustness Against Pose/Illumination Variation}

The robustness to expression and slight pose variations can be achieved by incorporating partialness in the measure. The robustness of the proposed transformation to illumination variation is illustrating during images from one of the testing databases. Fig. 2(a) and 2(b) are images of same person under different illumination and $2(\mathrm{c})$ is the image of adifferent person. The corresponding transformed images are3(a), 3(b) and 3(c).The error image for 4(a) and 4(b) is almost dark which shows that the transformed images are less affected by illumination variations. Ithas larger values over nose, mouth and eye regions and hence discriminate the transformed image for different faces.

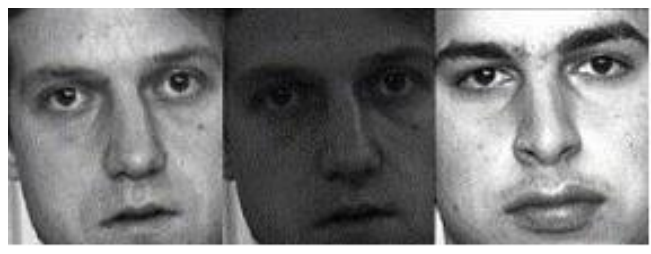

Fig. 2(a), 2(b) and 2(c) original images

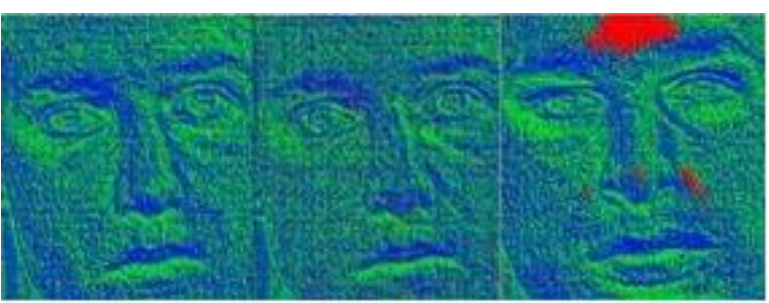

Fig. 3(a), 3(b) and 3(c) transformed images

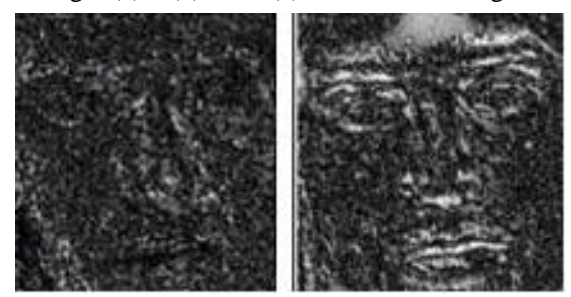

Fig. 4(a) and 4(b) error images

\section{Partial Hausdorff Distance}

\section{A. Definition}

Let $A$ and $B$ be the face of images of size $r \mathrm{x} \mathrm{c}$ to be compared. Let $A_{v}$ and $B_{v}$ are the transformed image. The border pixels of $\mathrm{M}$ and $\mathrm{T}$ are ignored for the transformed image as they do not have all eight neighbors. the size of $A_{v}$ and $B_{v}$ is therefore $(\mathrm{r}-2) \mathrm{x}(\mathrm{c}-2)$. Let $\mathrm{V}(\mathrm{P})$ represent the vector at pixel $P$. then the new hausdorff distance based measure between $\mathrm{A}$ and $\mathrm{B}$ is defined as

$$
H_{v}(A, B)=\max \left(h_{v}(A, B), h_{v}(B, A)\right)
$$

where $h_{v}$ is the directed version of $H_{v}$ and is given by

$$
h_{v}(A, B)=\underbrace{\max }_{a \in A_{v}} \underbrace{\min }_{b \in B_{v}} d(a, b)
$$

where,

$$
d(a, b)=\left\{\begin{array}{l}
\|a-b\|, \text { if } V_{a}=V_{b} \\
L, \text { if } V_{a} \neq V_{b}
\end{array}\right.
$$

The proposed measure can be improved for comparison application by introducing partial matching .Partial matching is obtained by taking $\mathrm{K}^{\text {th }}$ ranked distance instead of maximum in $\mathrm{h}_{\mathrm{v}}$. The equation are given by

$$
h(A, B)=\underbrace{K^{t h}}_{a \in A_{v}} \underbrace{\min }_{b \in B_{v}} d(a, b)
$$

The value of $\mathrm{K}$ chosen for this work is $0.5 \times \operatorname{size}(\mathrm{A})$.

\section{B. Computational Algorithm}

The computation of $h_{p v}(\mathrm{~A}, \mathrm{~B})$ involves finding the nearest pixel $b$ in $B_{v}$ for each pixel a in $A_{v}$ with $\mathrm{v}(\mathrm{a})=\mathrm{v}(\mathrm{b})$. Instead of scanning the entire image, if linked list of pixels in $B_{v}$ is constructed for every possible vector, then the search for the nearest pixel can belimited. There are 38 possible vectors and hence an array of 38 linked list are constructed. The elements of array are pointer to the linked list. The array index $i$ is computed from vector $\left[n_{0}, \ldots, n_{7}\right]$ as $i=\sum_{k=0}^{7}\left(n_{k}+1\right) \times 3 k$ the pictorial representation of the data structure is shown in Fig. 5.

Once the data structure is created for $B_{v}$, the computation of $h_{p v}(\mathrm{~A}, \mathrm{~B})$ is done as follow. For each pixel in $A_{v}$, the linked list corresponding to its vector value is search linearly for the nearest pixel and the distance to it is assigned. This leads to the assignment of distance value to every pixel in $A_{v}$ the $K^{\text {th }}$ ranked distance is the computed. This gives $h_{p}(A, B)$. Similarly, $h_{p}(A, B)$ is computed by creating data structure for $A_{v}$ and $h_{p}(A, B)$ is the maximum of two distance.

\section{Time Complexity Analysis}

The vectorization of image of size $r$ and $c$ can be done in a single scan and hence the time complexity of the generating vector image is $\mathrm{O}(\mathrm{rc})$. Index of the linked list array can be computed in constant time. Insertion of all the pixels in image to corresponding lists takes $\mathrm{O}(\mathrm{rc})$ time. As finding the nearest pixel involves linear search of a list of pixels, the time taken by this operation depend on the length of list, and this has to be executed $2 \mathrm{rc}$ times. Hence, the time required to find distance will be $\mathrm{O}(\mathrm{rc})$ where $\mathrm{d}$ is the length of largest list. To sort the $\mathrm{H}$ values to get $\mathrm{K}^{\text {th }}$ maximum is done by using quick sort which is of order $\mathrm{O}(\mathrm{rclog}(\mathrm{rc}))$ on average. But $\mathrm{d}$ ranges from 1 to $\mathrm{rc}$ in the best to the worst case. Hence the worst case complexity is $\mathrm{O}(\mathrm{rc})$.

\section{PERFORMANCE TEST}

\section{A. Face Database Used}

The ORL face database [5] from AT\&T Laboratories, Cambridge University was one of the databases used consisting subjects of 40 subjects ( 36 men and 4 women). A set of images taken over varied periods of time in two sessions for each person. Figure 5 shows sample image of some subject.

The other was the Yale face database [6] consisting of 15 subjects each having 11 images. The images have expression variations as well as illumination variations due to a light source positioned at right,left and center with respect to the face. Some of the images of a person are shown in Fig. 6. 


\section{B. Tabular Results}

The presented method when compared with others existing methods on the basis of pose and illumination variation reveals that it is the way ahead of any of them in terms of recognition rate.

\section{RESULTS AND COMPARISONS}

TABLE I: COMPARISON BASED ON EXPRESSION VARIATION

\begin{tabular}{|l|l|l|l|l|}
\hline Expression & PHD & LEM & M2HD & $\mathrm{H}_{\mathrm{pv}}$ \\
\hline Smiling & $88.88 \%$ & $78.57 \%$ & $52.68 \%$ & $100 \%$ \\
\hline Angry & $77.77 \%$ & $92.86 \%$ & $81.25 \%$ & $96.82 \%$ \\
\hline Screaming & $31.25 \%$ & $31.25 \%$ & $20.54 \%$ & $81.74 \%$ \\
\hline
\end{tabular}

Further work is also possible to improve the time of algorithm which right now is not suitable for practical application.

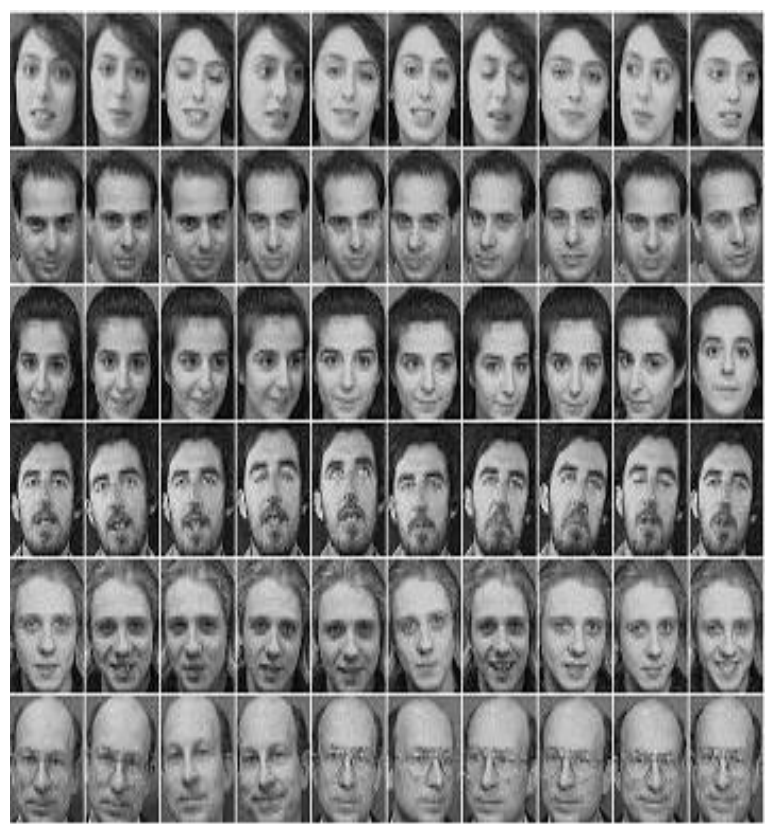

Fig. 5. Sample ORL face database

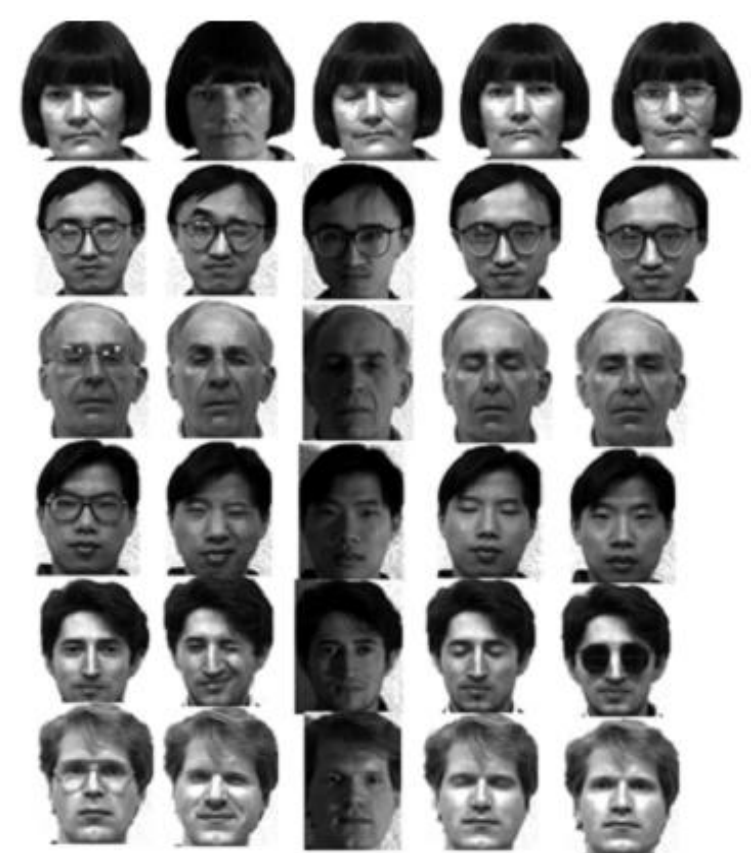

Fig. 6. Sample ORL face database
TABLE II: COMPARISON BASED ON POSE VARIATION

\begin{tabular}{|l|l|l|l|l|}
\hline Expression & PHD & LEM & M2HD & $\mathrm{H}_{\mathrm{pv}}$ \\
\hline Right/Left & $65 \%$ & $74.14 \%$ & $50 \%$ & $86.66 \%$ \\
\hline Up & $43.33 \%$ & $70 \%$ & $65 \%$ & $85 \%$ \\
\hline Down & $61.66 \%$ & $70 \%$ & $67.67 \%$ & $95 \%$ \\
\hline Average & $58.75 \%$ & $72.09 \%$ & $58.17 \%$ & $83.33 \%$ \\
\hline
\end{tabular}

TABLE III: RESULT FOR FIRST 5 BEST MATCHES

\begin{tabular}{|l|l|l|l|}
\hline $\begin{array}{l}\text { No. of Best } \\
\text { Matches }\end{array}$ & YALE & ORL(1-20) & ORL(21-40) \\
\hline 1 & $100 \%$ & $100 \%$ & $100 \%$ \\
\hline 2 & $100 \%$ & $98 \%$ & $100 \%$ \\
\hline 3 & $100 \%$ & $96 \%$ & $99 \%$ \\
\hline 4 & $100 \%$ & $89 \%$ & $93 \%$ \\
\hline 5 & $98.66 \%$ & $81 \%$ & $88 \%$ \\
\hline
\end{tabular}

\section{Time Taken a Function of Image Size}

Testing was done on LINUX based server (172.31.1.6) with single core 64-bits dual CPU (AMD opteron (TM) processor 250) machine with 8 GB RAM and $3 \times 140 \mathrm{~GB}$ hard disk. The test reveal following.

The algorithm is taking around 0.7-0.9 seconds per image for databases used where images are of the size $92 \times 112$ pixel in ORL and $75 \times 105$ in YALE database.

\section{DISCUSSION}

The results tabulated for this work seems to be batter that what is depicted in Vivek/Sudha et al. [7]. One reason for this could be the number of database used, which is laser (two against four) in this work. Secondly, care has been taken to keep the images of roughly the same size for YALE database used in this work and the ORL database gives nicely cropped and exactly the same sized image. Whereas in the Vivek/Sudha work, there is no mentioning of the cropping condition of image and their size. One way of removing image size dependence would be to scale up/down the images being compared so the distance are calculated on the same scale if the images happen to be of different sizes. Thirdly, the background in both databases use disconstant, but again there is no mention of the background in their work. It is seen that the time taken is a function of the number of pixels and increases just linearly with the number of images being compared. Further work can be done to improve the time taken by the algorithm. This can be done by pre-transforming the database images and also using some criteria to has them into groups. This would significantly reduce the matching time. One such criterion could be distance between the eyes or the relative position of the mouth with respect to the eyes etc.

\section{REFERENCES}

[1] K. M. Lam, K. H. Lin, and W. Chi, "Spatially eigenweighted hausdorff distance for human face recognition," Pattern Recognition Letters, vol. 36, pp. 1827-1834, December 2003.

[2] S. Srisuk and W. Kurutach, "New Robust hausdorff distance-based face detection," in Proc. Conference on Image Processing, 2001. vol. 1, pp. $1022-1025,2001$.

[3] B. F. Guo, K. M. Lam, W. Ch. Siut, and Sh. Y. Yang, "Human face recognition using a spatially weighted hausdofwf distance," in Proc. The 2001 IEEE International Symposium Circuits and Systems, ISCAS 2001, vol. 2, pp. 145 - 148, May 2001.

[4] R. Patel, N. Rathod, and A. Shah, "Comparative analysis of face recognition approaches: a survey," International Journal of Computer Applications, vol. 57, no. 17, pp. 50-69, November 2012. Published by Foundation of Computer Science, New York, USA.

[5] The orl database of faces, AT\&T Laboratories Cambridge . 
[6] Yale University Face Database. [Online]. Available: http://cvc.yale.edu/projects/yalefaces/yalefaces.html.

[7] E. Vivek and N. Sudha, "Robust hausdorff distance measure for face recognition," The journal of Pattern Recognition Society, vol. 40, pp. 431-442, April 2006.

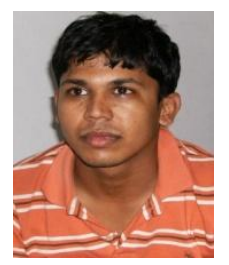

Chirag I. Patel is pursuing Ph.D. in Computer Science \& Engineering from Nirma Institute of Technology, Ahmedabad, Gujarat, India. He received B.E. in Information Technology Engineering from A.D.Patel Institute of Technology, New VallabhVidyanagar, Gujarat, India, in 2006, and the M.Tech. in Computer Science \& Engineering from Nirma Institute of Technology , Ahmedabad, Gujarat, India in 2009. His research interests are in video processing, statistical image processing and 3D vision.

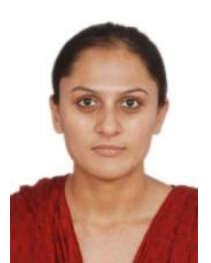

Ripal Patel obtained the B.E. degree in Electronics \& Communication Engineering from A.D. Patel Institute of Technology, New VallabhVidyanagar, Gujarat, India, in 2006, and the M.E. in electronics \& communication engineering from Dharamsinh Desai University, Nadiad, Gujarat, India in 2009. Her research interests are Computer Vision, Texture Classification, Video Processing and Image Registration. 